\title{
Slice-Aware Capacity Expansion Strategies in Multi-Tenant Networks
}

\author{
Özgür Umut Akgül ${ }^{\star \dagger}$, Ilaria Malanchini ${ }^{\dagger}$, and Antonio Capone ${ }^{\star}$ \\ ${ }^{\star}$ Dipartimento di Elettronica, Informazione e Bioingegneria (DEIB), Politecnico di Milano, Milano, Italy \\ Email: oezguerumut.akguel, antonio.capone@polimi.it \\ ${ }^{\dagger}$ Nokia Bell Labs, Stuttgart, Germany \\ Email: ilaria.malanchini@nokia-bell-labs.com
}

\begin{abstract}
G is envisioned to provide substantial increase in the network performance both in terms of achieved rate and delay. However, in order to satisfy these goals, the network operators are forced to make significant investments on the network infrastructure. Consequently a key question is how to invest the scarce economical resources to achieve maximum impact. Unlike the predecessor technologies, 5G contains a diverse mixture of services with unique requirements and priorities. From a network operator's perspective, solely focusing on the particular services makes it harder to determine the business value of a region. Therefore, in order to efficiently use the available expansion budget, the conventional approach of network dimensioning has be revisited. In this work, we propose a novel utility function based capacity expansion framework that can be automatized to dynamically and efficiently manage the infrastructure resources. Build upon three decision metrics, i.e. the urgency of the required resources, available investment budget and the expected impact of the capacity increase, our algorithm determines regions to invest in. Moreover, our analysis shows that the efficiency of the proposed framework does not strictly rely on the long term observation of the traffic demand.
\end{abstract}

\section{INTRODUCTION}

The lofty expectations posed by $5 \mathrm{G}$ require fundamental increase in the available network capacity. However, this intense network deployment places unprecedented economical strains on the network operators. From a broadband communication market perspective, the network operators with smaller market shares do not have the required revenue to expand the capacity resources, while the major operators who serve large portions of the market are unwilling to carry the economical burden of expansion due to the long return of investment duration. This economic pressure is forcing the network operators to reconsider their modus operandi in order to maintain a sustainable business model. Sharing the available network resources with other network operators is a promising solution to reduce costs [1]. In this new business model, wireless resources, which are provided by a third party, i.e. the infrastructure provider, are shared among a set of virtual operators, i.e. the tenants.

Moreover, 5G networks are envisioned to host a multitude of services and devices with unique requirements and service priorities. The traditional solution of optimizing the complete network for a particular service type is no longer applicable due to the conflicting requirements posed by different services.
A way out is to vertically group network resources (i.s. slicing the network) in order to create virtual dedicated networks per service. In this way, each slice can be customized to serve the respective service in the best possible way.

In the context of slicing and emerging technologies, one key aspect that has not yet been extensively investigated is the reinvestment of the collected revenues for capacity expansion. $5 \mathrm{G}$ technology gives rise to many business models and service types, each with demanding quality of service (QoS) expectations and priority. In this highly heterogeneous business ecosystem, determining the "value" of investing money in a given region is not clear. In addition to the long term strategies of the tenant, the value of a region is also a function of the service mixture in that region as well as the tenant's focus on particular services. Moreover, the traffic mixture variation over different regions makes it harder to understand the implications of the total QoS degradation on particular services and the urgency of the service needs [2]. Therefore, the conventional approach of investing in some regions only based on the experienced QoS is not necessarily the best possible strategy. Moreover, as detailed in [3], automated and fast network planning approaches are required to timely and efficiently shape the network resources in a multi-tenant scenario.

\section{A. Related Works}

Although the problem of capacity network expansion is usually investigated under several steps, the two key aspects are capacity dimensioning and planning. During the dimensioning of the network resources, decisions are made on deploying additional resources to different regions but without any particular decision on the exact location of the new resources, e.g. [3]-[5]. In contrast, planning phase determines where to locate the new resources based on radio maps, e.g. [6], [7]. The majority of the existing literature on capacity dimensioning do not consider the existing infrastructure resources and provide models for complete network deployments [8]. However, unlike the previous technologies, 5G deployment is not envisioned to replace the $4 \mathrm{G}$ infrastructure resources. Therefore, the network planning in $5 \mathrm{G}$ strongly requires awareness of the existing network infrastructure as well as the long and short term variations of the network traffic [9]. Moreover, the unique $5 \mathrm{G}$ aspects such as multi-tenancy and 
the heterogeneous service requirements have to be considered in the planning decisions. [3] provides a self-dimensioning algorithm for small cells in a multi-tenant network. However, their model relies on average QoS degradation and does not consider how different services perceive this decreased QoS.

Another challenge, covered in [10], is the fact that the network operators do not always possess the necessary economical revenue in order to upgrade or expand their resources to reach the maximum utility over all the coverage area. [11] provides a detailed cost analysis of network deployments, considering not only the device cost but also the respective deployment and capacity costs. A popular solution, which is not only used to handle the excess traffic in short term, but also for cases of public safety and disaster management, is deploying mobile base stations [4], [7].

\section{B. Contributions and organization of the paper}

A real time trading model of wireless resources is presented in [12], where we propose a novel market-driven pricing mechanism. Build upon our framework in [12], this paper proposes a novel capacity expansion model that guides the reinvestment strategies by assessing the value of expansion per region. Consequently, our key contributions in this work are as follows.

- We design a utility-based decision mechanism to compare the QoS degradation in different regions, taking into account service requirements and traffic mixture.

- We propose a novel self-dimensioning framework that is used to reinvest the aggregated economical revenues in an automated fashion.

- We demonstrate the advantages of short term investment decisions using a set of simulations based on real traffic data.

The rest of the paper is organized as follows. We present our system model and the key assumptions in Section II. Section III details the proposed mathematical model and presents our applied algorithm. The evaluation of our model is performed in Section IV. Finally, Section V concludes the paper.

\section{SYSTEM MODEL}

In this work, we use a similar notation to our previous work in [12]. As presented in Fig. 1, the total geographic area is given by $A_{c}$. For the sake of simplicity, we assume that $A_{c}$ is divided into a number of regions $R$, which are indexed by $r$ (cf. Fig. 1). The total area, $A_{c}$, is assumed to be totally covered by a number of base stations $B_{r}$ (shown as blue triangles in Fig. 1), namely there is no coverage gaps in the area. We assume that being the provider of the physical resources, the infrastructure provider is responsible for keeping the capacity resources in line with the demand. Consequently, the infrastructure provider decides on triggering the capacity expansion. The need for capacity expansion is decided within an observation period of $W_{\mathrm{ex}}$. The average number of users that are served by base station $b$, during the observation period is given by $K_{b}$.

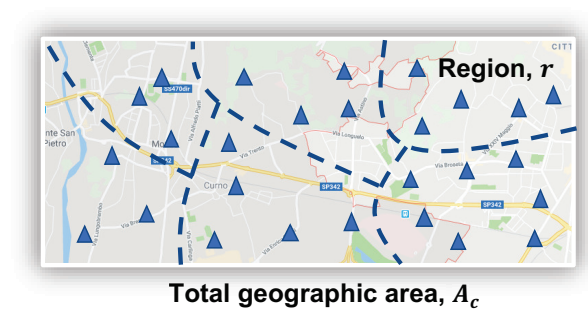

Fig. 1: The envisioned network topology

Similar to [12], the SLA between tenants and the infrastructure provider sets the utility goal per tenant, i.e. $U_{\text {th,m }}$ which defines each tenant's expected average total achieved utility. The difference between tenants' utility goals, i.e. $U_{\text {th,m }}$, and the actual achieved utility, i.e. $U_{k}\left(R_{k}, n\right)$, is referred to as the tenant's gap, $\xi_{m}$. The total gap of tenants and the collected revenue for capacity expansion are used to determine the urgency of the expansion decision over time. Note that the dynamic pricing framework, which is detailed in [12], is built upon a market-driven pricing strategy. In this model, the total cost of a tenant consists of three components. The first two terms are the operational and capital expenses, which are scaled according to the actual resource consumption and the guaranteed resource share of a tenant, respectively. The third term is the pressure cost, which is scaled proportionally to the tenant's gap $\xi_{m}$. During the observation period, the associated pressure revenues accumulated by the infrastructure provider can be written as

$$
R_{a c c}=\sum_{i \in W_{e x}} \sum_{m \in M} \xi_{m}[i] C_{\mathrm{pre}}
$$

where $C_{\text {pre }}$ is the unit pressure cost set by the infrastructure provider and $M$ is the set of tenants.

At the end of $W_{\mathrm{ex}}$, the capacity expansion decision is made based on the accumulated pressure revenue, $R_{\text {acc }}$, and the total gap per region, i.e. $\xi_{r}=\sum_{b \in B_{r}} \sum_{m \in M} \xi_{m}$. In case a tenant does not have any user in a particular base station, the respective utility expectation is set to zero, $U_{\text {th }}=0$, and consequently the gap of tenant $m$ in the given base station will be $\xi_{m}=0$. After modeling the available revenues for capacity expansion, the key challenge is how to model the cost for the additional resources. In this case, the considered resource type plays a key role for determining the cost of a resource. In this work, we use the cost model proposed in [11], which models the total cost of building a base station as an aggregation of equipment cost $\left(C_{1}\right)$, capacity cost $\left(A_{01}\right)$ and infrastructure cost $\left(B_{01}\right)$. Based on their proposed generic model that can be customized per technology, the cost of building an eNB can be calculated as

$$
C_{\text {tot }}\left(\lambda_{b}\right)=\lambda_{b} C_{1}+\lambda_{u} A_{01}+\frac{\lambda_{u}}{\lambda_{b} \pi} B_{01},
$$

where $\lambda_{u}$ and $\lambda_{b}$ indicates user and base station density, respectively. Since the deployment of the new eNBs are paid by the aggregated pressure cost during the observation period, 
the accumulated revenue $\left(R_{a c c}\right)$ has to be equal or greater than the cost of the newly deployed base stations. For the sake of simplicity, we assume that the densities are calculated for a unit area, so the cost of a base station is equal to $C_{\text {tot }}=C_{1}+\lambda_{u} A_{01}+\frac{\lambda_{u}}{\pi} B_{01}$ and the maximum number of base stations that can be deployed is $\lambda_{b}^{\max }=\left\lfloor R_{a c c} / C_{\text {tot }}\right\rfloor$. Depending on the observation window and the preferences of the tenants and the infrastructure provider, without loss of generality, (2) can be customized for the preferred expansion type.

\section{SELF-DimENSIONING FRAMEWORK}

Based on the calculation of $\lambda_{b}^{\max }$ proposed above, the problem of capacity scaling translates into determining the most efficient locations for capacity expansion. The efficiency of capacity expansion is measured in terms of decrease in the total gap in the region over a unit increase in the available capacity, $\frac{\partial \xi_{r}}{\partial \lambda_{r}}$. Since increasing the number of base stations decreases the experienced total gap in a region, we propose the following objective function for our problem

$$
\min \sum_{r \in A_{c}} \frac{\partial \xi_{r}}{\partial \lambda_{r}} \lambda_{r}
$$

Based on the correlation between the gap and the achieved utility, (3) can be rewritten as

$$
\min \sum_{r \in A_{c}} \frac{\partial \xi_{r}}{\partial R_{r}} \frac{\partial R_{r}}{\partial \lambda_{r}} \lambda_{r}
$$

where $R_{r}$ indicates the achieved spectral efficiency in region $r$. The first term, i.e. $\frac{\partial \xi_{r}}{\partial R_{r}}$, measures the change in the total gap of a region for a unit increase in $R_{r}$. By replacing $\xi_{r}$ with the gap definition, i.e. $\xi_{r}=U_{\text {th }, m}-U_{r}\left(R_{r}, n\right)$, we obtain

$$
\frac{\partial \xi_{r}}{\partial R_{r}}=\frac{\partial\left(-U\left(R_{r}, n\right)\right)}{\partial R_{r}} .
$$

The service heterogeneity envisioned in $5 \mathrm{G}$ is modeled using the quality of experience mapping that we proposed in [12]. In this study, we focus on four exemplary services whose utility functions are presented in Fig. 2a. The total utility function of a region, $U_{r}\left(R_{r}, n\right)$ is defined as

$$
U_{r}\left(R_{r}, n\right)=\sum_{b \in r} \sum_{k \in K_{b}} U_{k}\left(R_{k}, n\right),
$$

where $U_{k}\left(R_{k}, n\right)$ is the achieved utility of user $k$ at time slot $n$. Unlike our previous work, the proposed self-dimensioning framework is designed for relatively long time intervals (e.g. days, weeks or months). Consequently, the number of users in each base station and the traffic mixture dynamically change over time. As the network scheduler maximizes the total achieved utility, the regions with a negative value, presented in Fig. 2a, have the highest priority in our model. Namely the biggest priority of the scheduler is to provide resources to inelastic and machine to machine (M2M) traffic. Therefore, for the sake of tractability, we assume that all the base stations have a minimum amount of resources that is sufficient to satisfy this minimum resource requirements. Consequently,
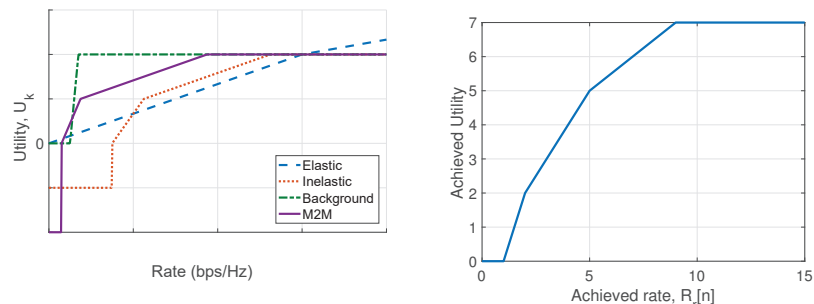

(a) Utility functions of the examplary (b) Examplary utility function of a service types

base station

Fig. 2: Framework's adaptability to the changes in the channel condition

the aggregated utility function (cf. Fig. 2a) does not have any underutilized region, i.e. the utility values below zero.

Substituting (5) in (4), our objective function becomes

$$
\max \sum_{r \in A_{c}} \frac{\partial U_{r}\left(R_{r}, n\right)}{\partial R_{r}} \frac{\partial R_{r}}{\partial \lambda_{r}} \lambda_{r} .
$$

In (7), the second term estimates the change of spectral efficiency over a unit increase in the base station density. However, the actual change in the spectral efficiency depends on the location of the new base station, therefore, accurately modeling the implications of a new deployment is very challenging. On the other hand, in [13], based on the homogeneous point processes, the expected value of the spectral efficiency is calculated by using

$$
R_{r}=\frac{\pi^{5 / 2}}{2} \sqrt{\frac{\lambda_{u} \lambda_{b} P}{\sigma^{2}}} \operatorname{Erfc}\left[\frac{\pi^{2} \lambda_{u}}{4} \sqrt{\frac{P}{\sigma^{2}}}\right] \exp \left[\frac{\pi^{4} \lambda_{u}^{2} P}{16 \sigma^{2}}\right],
$$

where $P$ shows the transmission power and $\sigma^{2}$ is the noise power. Furthermore, authors in [13] prove that if $\lambda_{u} \sqrt{\frac{P}{\sigma^{2}}}>>$ $\frac{4}{\pi^{2}}$, then (8) can be simplified to

$$
R_{r}=2 \sqrt{\frac{\lambda_{b}}{\lambda_{u}}}
$$

Thus, the second term in (7) can be rewritten as

$$
\frac{\partial R_{r}}{\partial \lambda_{r}} \equiv \frac{1}{\sqrt{\lambda_{r} \lambda_{u}}},
$$

and the complete objective function becomes

$$
\max \sum_{r \in A_{c}} \frac{\partial U_{r}\left(R_{r}, n\right)}{\partial R_{r}} \frac{\sqrt{\lambda_{r}}}{\sqrt{\lambda_{u}}} .
$$

Note that $\lambda_{r}$ is the only variable in our objective function, while $\lambda_{u}$ is a parameter which is calculated a priori by using $K_{b}$. In order to solve the proposed framework with a linear optimizer, without loss of generality, (11) is linearized as

$$
\max \sum_{r \in A_{c}} \frac{\partial U_{r}\left(R_{r}, n\right)}{\partial R_{r}} \frac{\lambda_{r}}{\sqrt{\lambda_{u}}} .
$$

The complete optimization model is given in (13.a)-(13.b), where (13.b) sets the upper bound for the number of deployed base stations. 


$$
\begin{gathered}
\max _{\lambda_{r}} \sum_{r \in A_{c}} \frac{\partial U_{r}\left(R_{r}, n\right)}{\partial R_{r}} \frac{\lambda_{r}}{\sqrt{\lambda_{u}}} \\
\text { s.t. } \quad \sum_{r \in A_{c}} \lambda_{r} \leq \frac{R_{\text {acc }}}{C_{\text {tot }}}
\end{gathered}
$$

However, to guarantee an accurate estimation of the locations where to expand the capacity, we enforce an iteratively deploy of additional resources, i.e., add one new base station at the time. Therefore, (13.b) is updated as

$$
\sum_{r \in A_{c}} \lambda_{r} \leq 1 .
$$

Finally, the self-dimensioning algorithm is implemented using Algorithm 1.

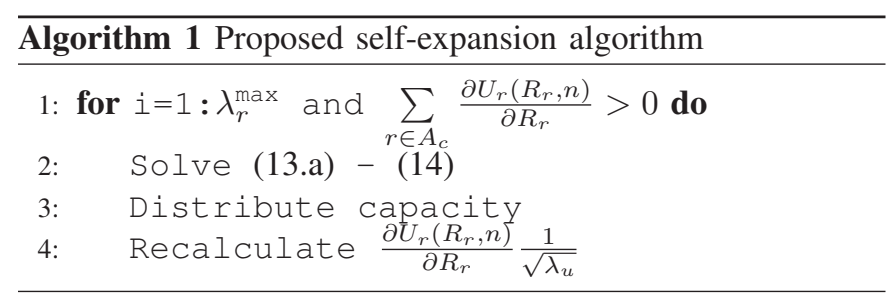

\section{Simulation Results}

\section{A. Traffic model}

In our simulations, we use the actual traffic demand collected from Bergamo, Italy. The data contains the total traffic demand over 39 base stations spanning the 12 months between March 2018 and March 2019. In the simulations, we focus on two main scenarios, i.e. the scenario where there are equivalent traffic distributions in each region (cf. Fig. 3a) and the asymmetric traffic distribution where the regions are divided based on the geographical distribution of the base stations, namely, residential, semi-residential, rural, industrial and state-facilities (cf. Fig. 3b). In the first scenario, the traffic mixture is assumed to be homogeneous, whereas in the second scenario it is assumed to be as given in Table I. Note that since the traffic data is used to measure the effectiveness of the proposed model, we assume that the average volume over one year and the cyclic characteristics do not change over the simulation horizon. This assumption is made to isolate the

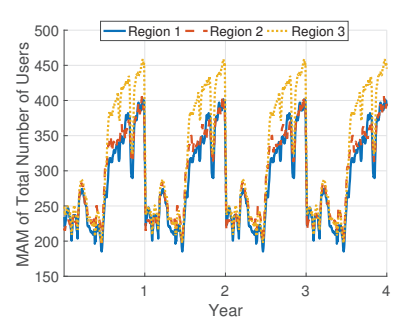

(a) Symmetric traffic distribution

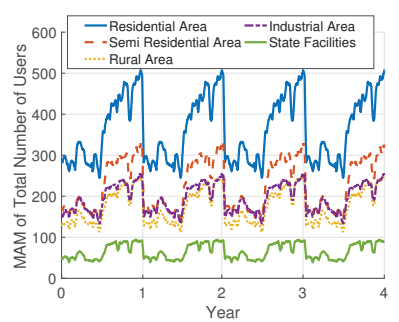

(b) Asymmetric traffic distribution
Fig. 3: Total number of users over time per region
TABLE I: The service distributions in percentage (\%) per region in scenario 2 .

\begin{tabular}{||c|c|c|c|c||}
\hline Region Type & Elastic & Inelastic & M2M & Background \\
\hline \hline Residential & 25 & 25 & 25 & 25 \\
\hline Semi-residential & 50 & 25 & 0 & 25 \\
\hline Rural & 25 & 0 & 0 & 75 \\
\hline Industrial & 25 & 0 & 75 & 0 \\
\hline State-facilities & 0 & 0 & 100 & 0 \\
\hline
\end{tabular}

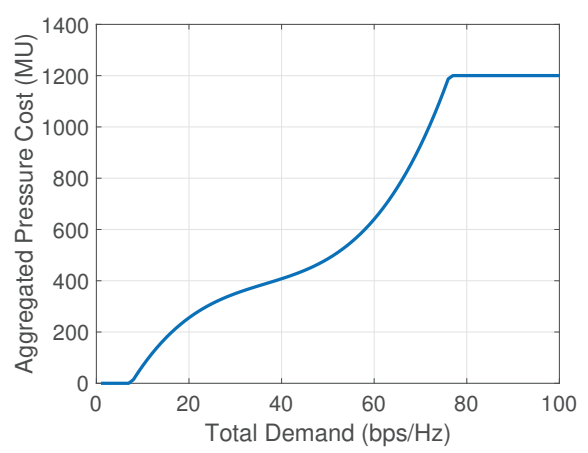

Fig. 4: Considered characteristic of the scheduler in [12]

effects of applied data set from the framework's characteristic. Thus, we have extended one year data into four years by copying the available data (cf. Fig. 3).

\section{B. Characterization of the real-time scheduler}

Built upon real time resource allocations and tradings, the proposed framework requires a set of observations on the average demand, the total gap and the respective pressure revenue. However, in order to accurately estimate the required capacity and collect a reasonable amount of revenue, the proposed framework requires relatively longer observation periods, i.e. in terms of days to months. Thus, in order to obtain the simulation results within an acceptable time, the sharing platform is modeled as a function of the number of users and the traffic mixture, as presented in Fig. 4. Namely, built upon a large set of simulation results, we generate a characteristic function that relates the number of users and the traffic mixture with aggregated pressure cost, i.e.

$$
f(x)=0.2854 x^{3}-57.7510 x^{2}+4496.5 x+51663 .
$$

Note that this function is purely designed for simplicity and it estimates the values that would be obtained in the application of real time trading model.

\section{Capacity evolution for symmetric traffic demand scenario}

An evolution of the proposed framework under the aforementioned first scenario (cf. Fig. 3a) is presented in this section. Observation window is chosen to be 1 month, i.e. $W_{\text {ex }}=1$ month. Fig. 5 shows the evolution of the total network capacity per region over the simulation horizon. Due to the inaccurate capacity, through the first year, we observe a very high capacity deployment phase. As a result of the 


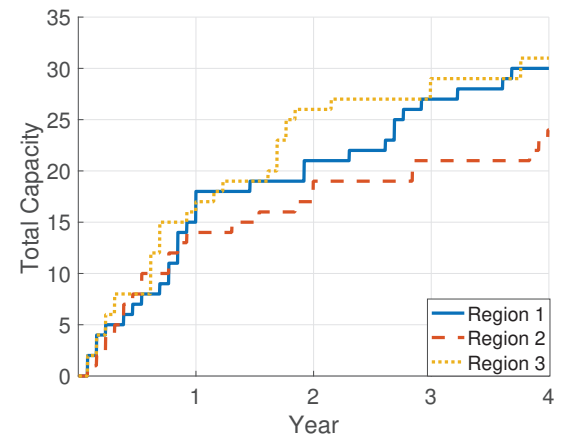

Fig. 5: The base station deployments per region

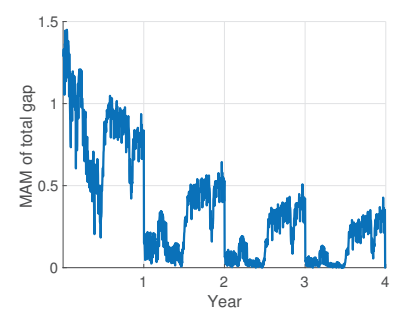

(a) The moving arithmetic mean of the total gap over all the regions

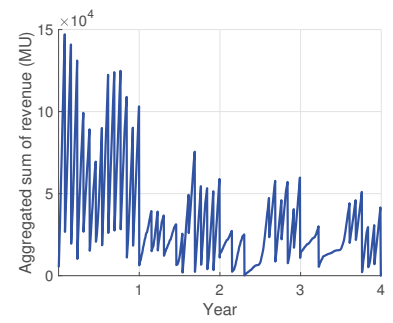

(b) Aggregated pressure revenue
Fig. 6: The evolution of the network performance

symmetric traffic demand and service mixture, during the first year, we see equivalent slopes in the capacity deployments over the regions. After this initial phase, the regions show small changes in their capacity evolution (cf. the second and the following years in Fig. 5), which is governed by the monthly variations in the traffic demand and the maximum number of base stations that can be deployed, $\lambda_{b}^{\max }$.

The evolution of the moving arithmetic mean of the total gap is given in Fig. 6a. The increasing network capacity causes a decrease in the total gap of the tenants. As an impact of the aforementioned deployment phase within the first year, the total gap shows a rapid decrease to nearly one third of the initial value. However, on the second half of the first year, as a result of the tremendous increase in the total traffic demand, the total gap also increases. Following the decrease in the total gap, a proportional drop in the total aggregated pressure revenue is also measured (cf. Fig. 6b). Especially during the first part of the fourth year, the collected revenue is sufficient to perform one capacity expansion. Consequently, the drop in the total gap gradually decreases over time, as the number of expansions decreases. However, as detailed in [12], this low level of gap is mostly affecting the elastic traffic. As a consequence of this lack of urgency and the low level of observed gap, the proposed framework distributes the capacity expansion process over time.

\section{Capacity evolution for asymmetric traffic mixture}

This section focuses on the aforementioned second scenario, where the regions are selected in terms of the service mixture. We group the base station using their locations, leading to

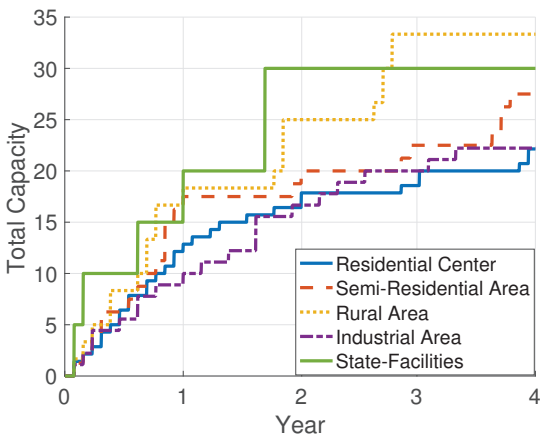

Fig. 7: The base station deployment per region

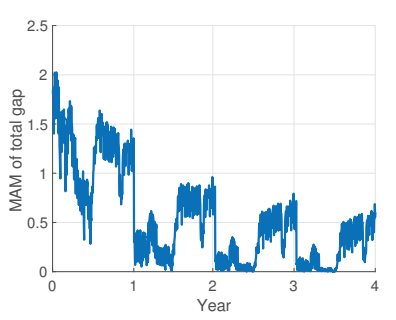

(a) The moving arithmetic mean of the total gap over all the regions

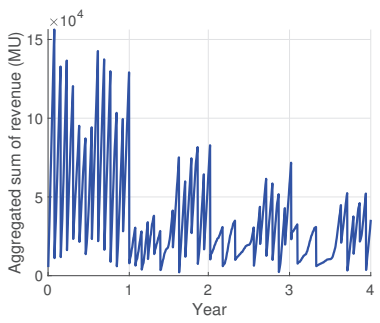

(b) Aggregated pressure revenue
Fig. 8: The evolution of the network performance

asymmetrical distributions of the number of base stations per region and the total traffic demand (cf. Fig. 3b). Fig. 7 presents the capacity evolution per region for this asymmetric scenario. The state-facilities (green line) indicates the region with the uttermost importance, as it contains the area with hospital and governmental entities, e.g. police. Consequently, in contrast to the observed low user density in the area, the proposed framework chooses to expand the resources in this region as the first place (cf. green line in Fig. 7). Note that despite our algorithm's focus on this aforementioned state-facilities, the proposed framework performs a certain level of investment to the rest of the regions as well.

Fig. 8 shows the aggregated total revenue over time (cf. Fig. 8b) and the evolution of the total gap given the reinvestment decisions of the proposed framework (Fig. 8a). Similar to the results outlined in Fig. 6, the increasing number of base station decreases the total gap. The main difference between Fig. 6a and Fig. 8a, i.e. the maximum measured value of the gap is a direct result of the two additional regions in this scenario. Moreover, similar to the symmetric scenario, for asymmetric scenario we can still observe that the proposed revenue collection mechanism guarantees efficient and timely capacity expansion (cf. Fig. 8b). More specifically, the transient conditions or fluctuations in the traffic demand do not affect the expansion decisions as they cannot accumulate the necessary revenue for a capacity expansion.

\section{E. Implications of the observation period length}

In order to provide a timely capacity increase, a critical aspect is the total observation period required for an efficient 


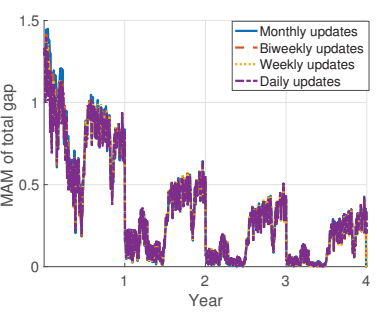

(a) Long time scale

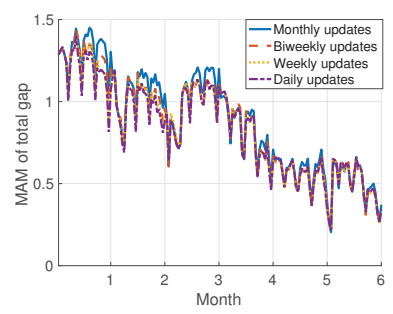

(b) Short time scale
Fig. 9: Implications of the observation period on the evolution of the network performance

expansion decision. In this section, we investigate the implications of using a smaller period than a month. Fig. 9a demonstrates the variations of the network performance evolution when changing observation period. The results show that in the long term, the observation period length has no visible impacts on the network performance.

Fig. 9b shows the short term (i.e. up to six months) implications of different observation windows on the network performance. On one hand, the shorter observation periods give the infrastructure provider less data and less money to use for the capacity expansion decision, on the other hand, the infrastructure provider has the flexibility to decrease the total gap faster since the collected revenue is almost instantaneously reinvested. In Fig. 9b, we observe that the daily updates (the purple dashed-dotted line) outperforms the monthly updates (blue line) due to early decision making mechanism. Consequently having a smaller observation period gives the infrastructure provider the chance to dynamically scale the available network resources. A through analysis of Fig. $9 \mathrm{~b}$ shows that in our proposed framework, at the end of each month the overall efficiency of the network is similar regardless of the length of the observation period. More specifically, after the capacity expansion at the end of each month, all methods reach to the same gap level, which shows that although the different observation windows can cause differences in the region selection, the expansion decisions always have equivalent impact on the total gap. This is a direct result of our utility based decision mechanism. Namely, even if two different regions are selected for the capacity increase, their impact on the overall achieved utility is the same.

\section{CONCLUSION}

The capacity management and reinvestment decisions are becoming overly complicated as the number of services sharing the same network increases. In order to preserve their business value, the network operators are required to have clear understanding on the business profile (e.g. service mixture) and the extra capacity need per region. In this paper, we propose a self-dimensioning algorithm for the reinvestment decisions that efficiently deploys new base stations according to the expected impact per region. Based upon the urgency of the capacity expansion, accumulated revenue and the expected impact on the total gap, the proposed framework can timely and accurately take capacity expansion decisions per region. Moreover, the proposed framework can use small observation periods (e.g. a day) without loss of efficiency. Therefore, the network resources can be scaled efficiently within relatively short time and in a profitable way.

\section{ACKNOWLEDGMENT}

This work is funded by the European Unions Horizon 2020 research and innovation programme under the Marie Sklodowska-Curie grant agreement No. 643002.

\section{REFERENCES}

[1] OECD, "Wireless market structures and network sharing," 2014 [Online]. Available: http://dx.doi.org/10.1787/5jxt46dz19r2-en

[2] A. Okic, A. E. C. Redondi, I. Galimberti, and F. F. amd L. Venturini, "Analyzing different mobile applications in time and space: a city-wide scenario," in IEEE Wireless Communications and Networking Conference (WCNC), April 2019. [Online]. Available: https://tinyurl.com/okic-wcnc2019

[3] P. Muoz, O. Sallent, and J. Prez-Romero, "Self-dimensioning and planning of small cell capacity in multitenant $5 \mathrm{G}$ networks," IEEE Transactions on Vehicular Technology, vol. 67, no. 5, pp. 4552-4564, May 2018.

[4] A. Georgakopoulos, I. Belikaidis, K. Tsagkaris, V. Stavroulaki, and P. Demestichas, "Wireless access infrastructure expansions through opportunistic networks of moving access points," in 2016 European Conference on Networks and Communications (EuCNC), June 2016, pp. $163-167$

[5] W. Ni, I. B. Collings, J. Lipman, X. Wang, M. Tao, and M. Abolhasan, "Graph theory and its applications to future network planning: softwaredefined online small cell management," IEEE Wireless Communications, vol. 22, no. 1, pp. 52-60, February 2015.

[6] N. Bhushan, J. Li, D. Malladi, R. D. Gilmore, D. Brenner, A. Damnjanovic, R. Sukhavasi, C. Patel, and S. Geirhofer, "Network densification: the dominant theme for wireless evolution into 5G," IEEE Communications Magazine, vol. 52, pp. 82-89, 2014.

[7] M. Mozaffari, W. Saad, M. Bennis, and M. Debbah, "Drone small cells in the clouds: Design, deployment and performance analysis," in 2015 IEEE Global Communications Conference (GLOBECOM), Dec 2015, pp. 1-6.

[8] G. D. Gonzalez, H. Hakula, A. Rasila, and J. Hamalainen, "Spatial mappings for planning and optimization of cellular networks," IEEE/ACM Transactions on Networking, vol. 26, pp. 175-188, 2018.

[9] J. Pérez-Romero, O. Sallent, R. Ferrús, and R. Agustí, "Artificial intelligence-based 5G network capacity planning and operation," in 2015 International Symposium on Wireless Communication Systems (ISWCS), Aug 2015, pp. 246-250.

[10] H. Setiawan, M. F. Rian Dinni, and D. A. Ratna Wati, "Lte network planning based on existing base transceiver using a genetic algorithm," in 2016 2nd International Conference on Wireless and Telematics (ICWT), Aug 2016, pp. 106-110.

[11] V. Suryaprakash and G. P. Fettweis, "An analysis of backhaul costs of radio access networks using stochastic geometry," 2014 IEEE International Conference on Communications (ICC), pp. 1035-1041, 2014.

[12] O. U. Akgül, I. Malanchini, and A. Capone, "Dynamic resource trading in sliced mobile networks," IEEE Transactions on Network and Service Management, 2019.

[13] V. Suryaprakash, A. Fehske, A. F. dos Santos, and G. P. Fettweis, "On the impact of sleep modes and BW variation on the energy consumption of radio access networks," in 2012 IEEE 75th Vehicular Technology Conference (VTC Spring), May 2012, pp. 1-5. 